\title{
Téoros
}

Revue de recherche en tourisme

\section{Le phénomène de mondialisation de l'industrie du tourisme}

\section{Thierry Quintrie La Mothe}

Volume 6, numéro 3, décembre 1987

Le tourisme, phénomène mondial

URI : https://id.erudit.org/iderudit/1080599ar

DOI : https://doi.org/10.7202/1080599ar

Aller au sommaire du numéro

Éditeur(s)

Université du Québec à Montréal

ISSN

0712-8657 (imprimé)

1923-2705 (numérique)

Découvrir la revue

Citer cet article

Quintrie La Mothe, T. (1987). Le phénomène de mondialisation de l'industrie du tourisme. Téoros, 6(3), 7-11. https://doi.org/10.7202/1080599ar d'utilisation que vous pouvez consulter en ligne.

https://apropos.erudit.org/fr/usagers/politique-dutilisation/ 


\section{Le phénomène de mondialisation de I'industrie du tourisme}

Un récent article d'un économiste américain attire l'attention sur un phénomène irréversible: la mondialisation des marchés. Selon Théodore Levitt ${ }^{(1)}$, deux vecteurs poussent le monde simultanément - le vecteur de la technologie et celui de la mondialisation. Le premier contribue puissamment à modeler les préférences humaines. Le second modèle les réalités économiques. La société mondiale moderne cherche constamment, "imperméble aux pressions et implaçable dans l'exécution", à imposer au monde entier des produits convenablement normalisés, choisis surtout quand des prix agressivement bas sont liés à lạ qualité et à la fiabilité.

Cette force puissante s'applique-t-elle aux. voyages et à l'industrie du tourisme? Assistet-on, dans ce secteur, auquel on prédit un grand avenir d'ici l'an 2000, à l'uniformité des besoins et des désirs des clientèles partout dans le monde et à l'homogénéisation des produits et des marchés? Quelle tristesse en perspective de voir notre planete se transformer en mille "Disney lands" ou pares récréatifs à péage, à défaut d'avoir su mettre ă temps "notre âme en lieu sûrr" et valoriser une authentique identite culturelle! La concurrence entre les destinations touristiques est de plus en plus vive. $\hat{A}$ l'image des chaines de TV, la lutte est sans pitié et malheur aux projets de "développement" qui ne correspondent pas aux exigences de la rentabilité financière. L'entreprise touristique devient-elle mondiale et cherche-t-elle, à son tour, à satisfaire des désirs uniformes et standardisés?

\section{La mondialisation du tourisme à l'épreuve}

Le tourisme est devenu en trente ans, de 1950 à 1980, un phênomène mondial. Tous les experts s'accordaient à penser que la croissance était exponentielle. Jusqu'en 1982, les chiffres leur donnaient raison: de 90 millions de touristes en 1963, nous sommes passes à près de 300 millions d'arrivées en 1984 faisant fi de la récession économique et du quadruplement du prix des produits pétroliers. La dynamique des déplacements de personnes n’allait-elle pas conduire à une intégration constante et progressive du monde?

Pourtant, des 1979 on observe un ralentissement de la croissance des dépenses des touristes. A la periode des "trente glorieuses" (1950-1980), succéde la période 1981-1985 caracterisée par la "tendance globale d'une moindre croissance des flux réels et financiers" cachant "des situations nationales extrêmement contrastées et une activité plus sensible aux à coups conjoncturels" ${ }^{(2)}$.

Quasi stagnation de 1980 à 1984 des arrivées de touristes, voire régression du nombre des touristes selon l'Organisation Mondiale du Tourisme; dépenses de voyages en baisse de 5 milliards de dollars entre 1980 et 1982, trafics passagers de $2 \%$ en taux de croissance annuel moyen entre 1980 et 1982 , alors qu"il était de $8,4 \%$ entre $1970-1980$.

La prudence $\mathrm{s}^{+}$impose. Selon P. Mallon, chargé de mission à la direction des industries touristiques, "la brutalité de cette évolution récente, certes contrastée (en particulier dans la zone Pacifique) amène aujourd'hui à accepter avec une extrême circonspection les prévisions genéralement établies par simple extrapolation des tendanoes. passées ou se fondant sur l'existence d'une corrélation trop étroite entre le tourisme et l'économie mondiale" "(3),

Néanmoins, un consensus semble se dégager entre les constructeurs aériens, selon la revue Futuribles, pour pronostiquer une croissance annuelle d'environ $6 \%$ par an pour le trafic international de 1985 a 1995 . Ceci correspond de toute façon à un ralentissement par rapport à la période précédente et une croissance relativement moins rapide pour les flux interrégionaux. La montée en puissance de la zone Pacifique se maintiendra comme zone émettrice et selon l'O.M.T. l'Europe pourrait conserver $70 \%$ du marché mondial des arrivées de touristes.
L'internationalisation du tourisme se présente par un lent déplacement des flux, selon les directeurs du marketing de la Commission Européenne du Tourisme (C.E.T.):

a) $1975-1985$

Zone émettrices

- Montée des pays pétroliers

- Maintien du couple USA-RFA

Flux interrégionaux

- Maintien de la position leader du marché transatlantique

b) 1985-1995

Zones emetrices

- Incertitudes concernant les "nouveaux émetteurs"

- Émergence des géants démographiques des NPI (nouveaux pays industriels)

Flux internationaux

- Renforcement des courants transPacifiques et Asiatiques

- Incertitudes concernant les marchés secondaires (Moyen-Orient, Afrique, etc...)

Au niveau international, on assiste aux effets contradictoires de deux logiques parallèles.

i) La logique de la concentration, c'est-à-dire:

standardisation des produits, recherche des économies d'échelle, osmose ProduitFonction.

ii) La logique de la diversification, c'est-ă-dire:

remise en cause des "Grands Projets", éclatement de la demande, déréglementation, pour le trafic aérien et la prestation de services, développement de la "tourismatique"".

Ces évolutions pourront favoriser une concurrence plus qualitative: redéfinition du positionnement stratégique, conception élargie du "métier" (vocation-identité), coopération tous azimuts, etc. (Source: Association Internationale Futuribles)

\section{La mondialisation}

\section{des producteurs de voyages}

De plus en plus, quelques entreprises touristiques recherchent une dimension mondiale en intervenant à tous les stades de la production (transport, hébergement, animation, ...) Elles semblent avoir le pouvoir d'orienter la demande mondiale par la création d'images de marque. Leur contrôle sur l'information 
TABLEAU 1

Nombre d'hôtels associés à de grandes sociétés transnationales à l'étranger

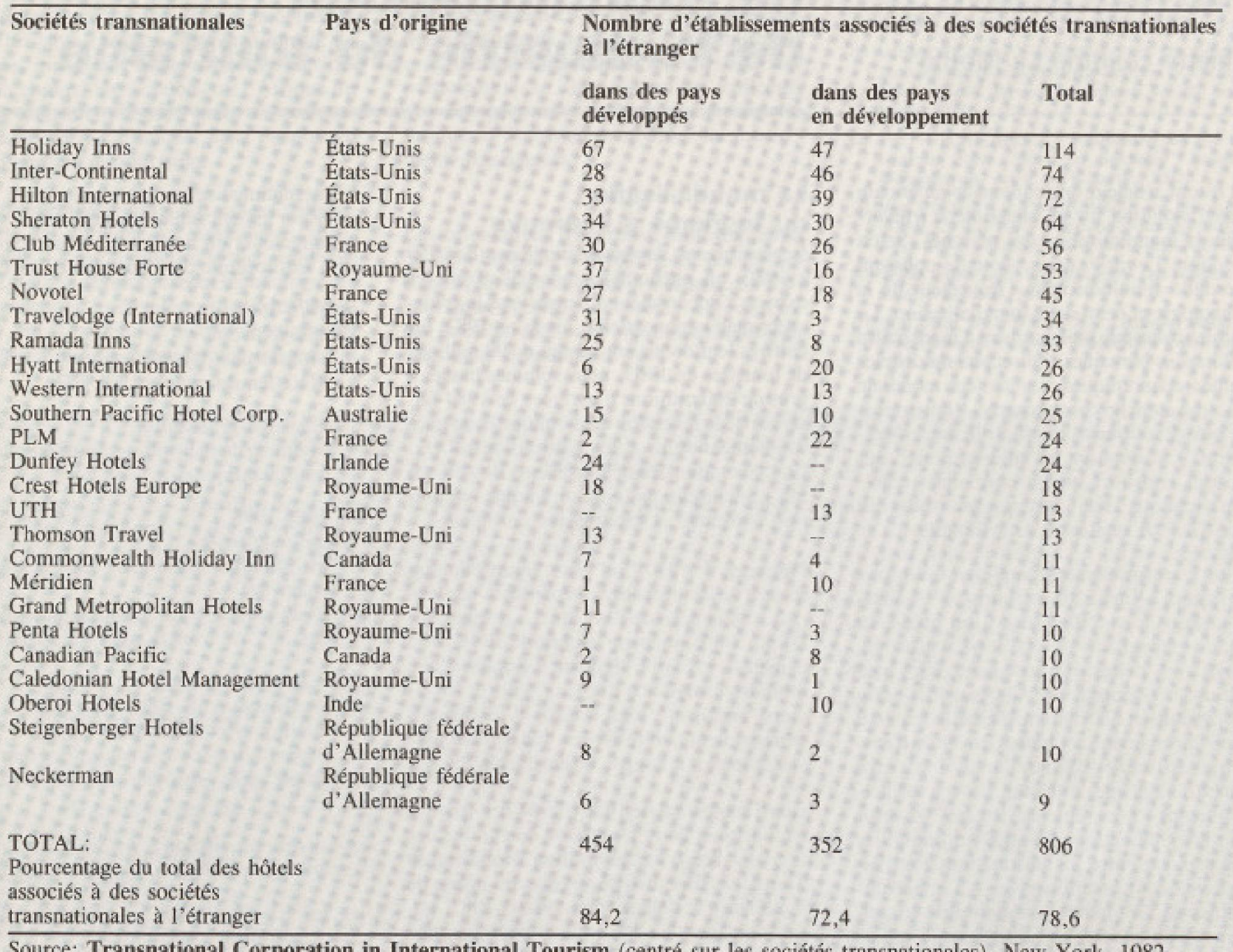

Source: Transnational Corporation in International Tourism (centré sur les sociétés transnationales), New York. 1982.

se renforce par l'extension des réseaux télématiques auxquels elles ont un accès privilégié, ce qui leur permet d'imposer leurs objectifs de gestion partout où elles implantent des filiales.

D'après les chiffres du Centre des NationsUnies sur les sociétés transnationales en 1982 (cf tableau no 1), 26 sociétés dont 16 étroitement liées à des compagnies aériennes, contrôlaient des hôtels représentant $34 \%$ de la capacité internationale des pays en développement. Quelquefois, la chaine hôtelière fait partic d'un ensemble plus vaste. Sur les 81 groupes hôteliers transnationaux dénombrés en 1982, neuf seulement étaient originaires de pays en developpement et un seul se classait dans les 26 sociétés précitées.

Les divers processus de rachats, fusions, créations de filiales et mises en commun de services divers tendent à donner à ces chaînes intégrées un profil identique et des stratégies de développement analogues.
La technique utilisée pour la conquète des marchés extérieurs consiste également à mobiliser un minimum de capital pour contrôler le plus grand volume d'affaires possible.

Paradoxalement, avec les progrès de l'ingénierie, de l'informatique et de la télématique, les transferts ne se réalisent plus. Seules quelques applications sont mises à la disposition des pays récepteurs pour qu'ils puissent s'insérer en tant qu'utilisateurs dans le réseau mondial de l'information et de la commercialisation.

La concentration mondiale des moyens de production et de commercialisation des biens touristiques, faworisée par l'innovation technique, a tendance à marginaliser les pays récepteurs qui n'ont plus accès à l'information. Donc, ils contrôlent une fraction de plus en plus faible de la demande internationale et bénéficient très peu de revenus produits par les mouvements internationaux de personnes.
Aux Êtats-Unis, le systeme de réservation electronique Apollo de United Airlines, accueille aussi la KLM, TWA et Delta Airlines.

En France, le système Alpha 3 regroupe Air France, Air Algérie, Royal Air Maroc, Air Afrique, Sabena, Tunis Air et UTA. Sous le contrôle d'Air France, il permet de réserver des places à partir de 3500 points d'accès dans le monde et est relié à 1700 hótels, aux organisateurs de voyages, des compagnies et aux agences de location de voitures.

Contrairement à une idé reçue, le coût d'emploi de ces systèmes n'est pas exhorbitant.

Ainsi, la mondialisation s'applique à certains eléments constitutifs du produit touristique, le transport par exemple. Phénomène irréversible, au-delà des politiques protectionnistes des Etats-Nations. Par exemple, au mois de mai 1987, les compagnies européennes, Lufthansa, Air France, la SAS et Ibe- 
ria se sont associées pour créer un réseau de réservation électronique à l'échelle mondiale afin d'empècher les compagnies américaines de monopoliser les agences de voyages et donc la clientèle du Vieux-Continent.

Le monopole du savoir-faire et de l'information au niveau du transporteur a pour résultat de canaliser la demande. Les voyageurs seront de moins en moins nombreux à choisir librement leur mode de consommation et à avoir un comportement économique actif et critique à l'égard des prestations qui leur seront proposées. Enfin, le système de paiement par carte à mémoire a pour effet d'acheminer les recettes touristiques vers les pays où les societés internationales ont leur siège.

Ainsi, la tendance générale est claire: la marge d'initiative des pays en développement est relativement réduite. La "tourismatique" devient un outil de compétition. "Elle s'exercera d'abord entre les biens de consommation et d'équipement d'une part et les services d'autre part. Elle se manifestera de plus en plus entre les secteurs professionnels, entre les entreprises, entre les régions, entre les pays" "(4).

L'accroissement du mouvement des personnes attendues dans le monde d'ici à l'an 2000 , amène à penser que "seuls les pays qui auront su prendre possession des réseaux, y imposer leurs notions de transaction afin d'y faire circuler leurs produits seront les gagnants de cette compétition" ${ }^{\prime(5)}$.

Les vieux pays européens seraient-ils condamnes à n'exploiter qu'une ressource pour maintenir un certain niveau d'emploi et d'exportation et à se transformer en "réserves touristiques". Dans un tel contexte, "l'organisation tourismatique", définie comme l'ensemble des démarches et outils de l'informatique et de ses dérivés au service des industries du tourisme et des loisirs, ${ }^{4}$ apparait comme un facteur déterminant de la concurrence entre pays récepteurs de touristes ${ }^{n(6)}$

On assiste à une concentration progressive des entreprises d'organisation de voyages.

En France, les 5 premiers tours opérateurs (Club Méditerranée exclu), assurent $46 \%$ de la production totale en 1984 contre $34 \%$ en $1981^{(7)}$

L'exemple du Club Méditerranéce est significatif du phénomène de mondialisation des producteurs de voyages.

\section{Le cosmopolitisme du Club Méditerranée}

Le Club Méditerranée est un des symboles de l'apparition de la société mondiale dans sa puissante réalité. Actuellement, le "Club $\mathrm{Med}^{\text {" }}$ vend son produit, uniforme et standardisé, aussi bien aux Européens qu'aux Américains ou aux Japonais. Le slogan "le Club antitode de la civilisation " est, à quelques nuances près, le mềme depuis près de 40 ans. Il s'agit toujours de proposer aux

TABLEAU 2

Évolution de la fréquentation des clientèles au Club Méditerranée entre 1977 et 1985 (en \%)

\begin{tabular}{lll}
\hline Clientèle & $\mathbf{1 9 7 7}$ & $\mathbf{1 9 8 4 / 1 9 8 5}$ \\
\hline Française & $47.1 \%$ & $40.9 \%$ \\
Allemande & 3.8 & 4.6 \\
U.S.A. & 17.9 & 23.8 \\
Australie & 0.6 & 1.2 \\
Japon & - & 1.9 \\
\hline
\end{tabular}

Source: Rapports d'activités du Club.

vacanciers une organisation des vacances où adultes et enfants trouvent une gamme d'activités et de distractions en toute sécurité. Le prix à payer est relativement cher. Qu'importe!

L'efficacité ne consiste-t-elle pas à intégrer une qualité et une fiabilité supérieures pour le prix offert? Le Club s'internationalise régulièrement. En 1984-1985, le nombre d'adhérents européens s'est accru de $4,28 \%$ mais il ne représente plus que $64,6 \%$ des adhérents de l'ensemble du Club $(67,4 \%$ en 1983-1984). L'internationalisation du groupe s'étend aux villages européens $(+10,6 \%$ d'Allemands, $+47,9 \%$ d'Anglais).

En dix ans, le Club Méditerranée est passé d'une stratégie transnationale (faire connaître ses villages à la clientèle française) à une véritable politique mondiale (remplir les formes d'hébergement de clientèles tous azimuths).

La lecture des chiffres du tableau ci-dessous (tableau no 2), montre bien la baisse du pourcentage des Français dans la fréquentation globale et par contre, l'augmentation du nombre d'Américains et la percée des pays du Pacifique.

Aux USA, les agents de voyages sont désormais reliés à un réseau informatique qui leur permet d'entrer en communication directe avec les systèmes de réservation du Club et d'enregistrer instantanément les inscriptions.

Le cosmopolitisme du Club lui permet, grâce à son image, de se greffer à toutes les expériences nouvelles de loisirs urbains ${ }^{(8)}$. Sa présence au Japon pendant une exposition internationale à Tsukuba a permis de faire découvrir aux visiteurs la vie d'un village de vacances et de manifester son implantation, comme une "place internationale de la communication".

Le Club Méditerranće n'est pas la seule organisation française à sंorienter résolument vers la mondialisation des marchés: il y a aussi le groupe Accor, la compagnie des Wagons lits, la chaine Méridien. Souvent les chaînes anglo-saxonnes (Hilton, Hyatt, Trust Houses) offrent le même produit à une clientèle mondiale (1'homme d'affaires indiquant des besoins standards facilement identifiables). Par contre, la chaine Méridien développe avec succes. "I'art de vivre Français à l'étranger". La petite touche nationale reste discrète, et la mondialisation n'exclut pas un petit effort d'imagination aux couleurs du pays hôte.

Ainsi, les voyages sont mondialisés comme les Mac Donald's, Coca Cola, Pepsi, la salade grecque dans le quartier latin à Paris, les "séries" B d'Hollywood et la musique... rock (la fameuse sono mondiale). Le plus petit Office de la France profonde rêve de "vendre son produit" en Chine, au Pacifique, aux îles Kergelen pourquoi pas? La prodigieuse vitalité des transactions financières et boursières entraîne dans son sillage toute une armée de candidats à la vente de leur terroir.

\section{La mondialisation de la forma- tion professionnelle}

Le développement des nouvelles technologies de la communication va-t-il bouleverser l'emploi dans l'hôtellerie et le tourisme? Télématique, vidéodisque, enseignement assisté par ordinateur, câbles, nouvelles images... I'entreprise touristique découvre de nouveaux outils pour satisfaire ses besoins. de communication interne et externe.

Le rapport Fast ${ }^{(9)}$, prévoit les créations de 4 à 5 millions d'emplois autour des nouvelles. technologies à l'horizon 1995 dans la CEE. Les profils des emplois et des formations correspondantes restent flous. Rien sur les nouveaux métiers ou les nouvelles fonctions tant espérées.

Les investissements dans la formation font partie des investissements de productivité analogues à ceux qui concernent les innovations technologiques. Si l'on considère la répartition des charges de fonctionnement, il apparait que l'accroissement des compétences constitue un facteur important de l'amélioration des performances de l'entreprise touristique, dans un domaine d'activité si peu normalisable et si résistant à la standardisation.

Les métiers de l'hôtellerie et du tourisme sont extrêmement différents. On a pu en dénombrer 300 de compétences diverses, rien que pour les services de l'hébergement. Quoi de commun entre la standardiste et le commis de cuisine? Métiers éminemment évolutifs, longtemps inchangés. En quelques années, les voilà pris dans le tourbillon de l'innovation technologique, des changements écono- 
miques, des mutations culturelles. Que d'aléas en perspective, que les recettes traditionnelles ne permettent pas toujours de résoudre! Il suffit de voir la préparation industrielle de la nourriture et son offre généralisếe sur le marché se substituant à la préparation domestique rituelle des repas, ou la diminution de la part "hébergement" dans la recette hôtelière au bénéfice d'activités de congrès, de réunion, qui exigent des prestations tout à fait spécifiques et intègrent nécessairement au personnel de l'accueil hôtelier. a des niveaux divers, des operateurs nouveaux. Nouveaux, mais travaillant dans lä même entreprise et susceptibles par conséquent de recevoir des ćléments de formation.

Cet aspect évolutif des métiers pose à l'appareil de formation un problème à la fois d'efficacité et de morale: comment éviter d'enseigner ce qui est ou risque d'être dépassé? Et comment déterminer et inclure dans les programmes, les innovations technologiques et les changements de mentalité.

\section{La fête se mondialise}

Pour un soir, Louxor en Egypte, est devenu le centre du monde, grảce à l'opéra. Comme du temps où elle était encore "Thebes aux cent portes", capitale des Pharaons. La représentation " $d$ 'Aïda" a a coutté quelques 10 millions de dollars, que le promoteur n'est pas sûr de pouvoir récupérer. De tels événements se répéteront...

Les nouvelles formes de loisirs pararécréatifs ou à thème mobilisent des capitaux importants pour atteindre une dimension mondiale. Musées, Festivals, maisons de la musique, golfs, parcs d'attractions et aqualands se multiplient pour le meilleur et le pire... Ces nouveaux concepts de loisirs sont orientés vers des besoins standardisés et adaptables à une consommation de masse. La rentabilité n'est guère assurée avec le risque de ternir l'image générale de ces nouveaux Équipements.

Le concept d'ingénierie culturelle a été inventé récemment. Aider une municipalité, une région, un pays à définir sa programmation et sa stratégie commerciale.

Il ne s'agit pas uniquement des parcs, golfs et autres "découvertes", mais bien "d'un nouveau regard sur le phénomène des loisirs et de la mise au point de méthodes d'analyses et de projections qui ont plus à voir avec la communication globale, la stratégie et la culture au sens large's(10)!

La culture mondiale devient une marque de commerce. Ce dénominateur commun du succès de toute fête par exemple, combine 3 elléments:

- un produit unique qui fait appel à la créativité et à l'innovation;

- offrir à son public une expérience personnelle enrichissante;

- faire place à la découverte en sortant des sentiers battus et des habitudes.
La culture, au sens large, devient un événement de plus en plus "tourne" vers l'extérieur. Un carnaval, comme celui de Rio, au Brésil, injecte dans l'économie locale près de 80 millions de dollars. Cette fête issue des forces "internes" d'une collectivité, de son imaginaire le plus intime est devenue un événement mondial. Même si les "bénéfices" engagés se font au détriment des principaux artisans de la fète.

\section{Les dangers de la mondialisation du mouvement des personnes}

Depuis 1976, la population s'est accrue de 700 millions d'âmes. Dans le même temps, les pays du Pacifique, en Amérique du Nord et en Asie ont créé plus de 55 millions d'emplois.

Les nouveaux pays industriels deviennent les nouveaux pays de l'intelligence (Taiwan, Corée du Sud, Hong-Kong, Singapour, Thaïlande, Inde, ...). $37 \%$ de l'effort de recherche japonais est investi en technologies de l'information. L'Europe n'y consacre que $17 \%$, "chaque entrepreneur des nouveaux industriels est habité par la conviction intime qu'il gagnera s'il applique correctement les concepts relatifs aux économies d'échelle, aux seuils critiques, à la concentration des moyens, à la mondialisation des marchés et $s$ 'il sait jouer avec la multinationalitét(11),

Mais le cadre touristique actuel semble trop restreint, face à la fulgurance du développement des outils d'information et de communication. La notion même de "vacances" semble remise en cause, sous son aspect traditionnel "congés payés". Les touristes se ramassaient à la pelle pensait-on. Il suffisait de les glaner. Éventuellement de les servir. La remise en cause des formes actuelles de déplacement n'empêche pas l'irréversible aspiration des personnes à inventer des formes nouvelles de rencontre et d'activités ludiques.

Dans un contexte historique, il convient de voir que les déplacements de personnes ne sont pas essentiellement constitués par des vacanciers, mais bien par d'autres voyageurs qui répondent à des innovations infinies. Le voyage en soi, la foi en une religion, les jeux, les compétitions, la santé par les cures thermales, les affaires, le commeroe, le sport, la culture, les etudes... Autant de motifs de déplacement qui se complexifient, et s'organisent, à l'échelle mondiale pour créer des emplois et des marchés de loisirs nouveaux.

La technologie moderne ne peut qu'accroître les échanges et les mouvements de personnes. La société mondiale, telle que la décrit Théodore Levitt, est effectivement en marche. La route poursuivie jusqu'ici par les voyageurs n'a fait qu'ouvrir l'avenir. Plus que l'objet de la destination finale du voyage, c'est le voyage lui-même qui doit être pris en considération, car c'est tout au long du voyage que le voyageur nécessite une série de services pour satisfaire son attente. Dès lors, il convient de souligner que le calcul économique qui mesure le succès de développement des voyages en termes de recettes en devises étrangères, sans se soucier du nombre d'emplois créés et de la qualité des services rendus est tout à fait inadéquat, car il signifie une approche statique au problème du développement dans les pays réceptifs. Une approche plus dynamique consisterait prócisément à concevoir les mouvements non migratoires sous l'angle des besoins et des réactions des personnes qui voyagent.

Tout voyage résulte de la combinaison de 4 éléments complémentaires et indissociables:

- l'envie de voyager, grâce aux média et surtout à la presse;

- le façonnage du produit;

- le transport, c'est-à-dire l'acheminement du voyageur;

- 1'accueil à la destination.

Le voyage devient un art, une véritable alchimie, un travail de haute couture qui n'exclut en rien le prêt à porter. Mais l'erreur serait de généraliser lạ démonstration d'un auteur comme T. Levitt à un secteur", celui des voyages, qui justement, ne supporte aucune approche globalisante et réunit un ensemble complexe de paramètres et de variables totalement aléatoires. Rien de plus erronées que les prévisions de fréquentation touristique! La mondialisation ne pourrait s'appliquer à la rigueur qu'à deux eléments, le façonnage du produit et le transport, mais très peu aux autres, qui restent subjectifs et déterminés par des influences difficilement comptables (la publicité, la mode... la solitude).

On ne peut nier l'existence de pays dominants et de pays dominés sur le plan touristique. La puissance des voyagistes allemands sur le bassin méditerranéen et celle, croissante des Japonais sur le Pacifique est impressionnante, imperméable aux pressions et implaçable dans l'exécution. "Loin de gommer les réalités nationales, le développement touristique a l'échelle mondiale les accentue, renforçant à un pôle des capitalismes nationaux dominants, utilisant à l'autre l'existence d'États-nations plus ou moins fantomatiques ${ }^{t+(12)}$. La domination Economique, financière et culturelle du capitalisme atteint, de nos jours, un degré très élevé d'extension et d'intensitế; jamais l'indépendance économique n'a autant été, pour la quasi-totalité des pays, un leurre. Au-delà de ce constat désabusé, les pays dominés peuvent non pas rêver à une "déconnexion nationale ${ }^{+"}$, mais reconquérir une marge de manoeuvre, favoriser une riposte diversifiée à tous les niveaux.

L'industrie des voyages se prête à ce travail de finesse et d'intelligence, que trop de penseurs, ennivrés par leur propre discours euphorique et généralisateur, considèreront, sans doute, avec une moue de dédain.

Les théoriciens de la "mondialisation des marchés" minimisent l'impact des minorités et des phénomènes marginaux, qui - en matière de voyage - sont pourtant déterminants. La planète qu'ils décrivent ne devien- 
drait qu'un paysage uniforme, gommant les contrastes. L'identité culturelle des pays est exclue. Les lois de la concurrence, le développement des moyens techniques, l'organisation des loisirs de masse banalisent toute la gamme des produits touristiques, tel le rouleau compresseur.

Les dangers de la mondialisation sont évidents. Plus elle "massifie" "le voyage, plus le voyage se cherche ailleurs que dans la masse. Mais s'il se trouve dans l'isolement, est-il encore Tourisme?

\section{La nécessité d'un certain désordre}

La création d'entreprise dans le tourisme devient un marché. On reconnaît dé plus en plus le rôle très important des petites et moyennes entreprises dans l'économie, mais on se laisse séduire aux pièges de la production de masse.

Mème dans le tourisme, la sécurité est plus considérée que l'aventure et l'enseignement conforte cette attitude: un jeune sur deux (de dix-huit à vingt-cinq ans) souhaite travailler dans l'administration.

Si la mutation d'ensemble est si lente, c'est parce qu'elle est essentiellement culturelle.

"Remettre les cerveaux en marche" n'est pas une mince affaire, indique Xavier Gizard dans un récent ouvrage sur la nouvelle entreprise ${ }^{(13)}$. L'important est de savoir que l'on ne peut plus isoler les facteurs de mutation, que l'évolution du tourisme dépend de celle de l'habitat, de la trame urbaine ou rurale, de l'aide à l'innovation, des profils de la population et de sa formation.

\section{L'intelligence peut aussi séduire le touriste}

Plus il y a de "produits" touristiques sur le marché mondial, moins il y a de choix. La gamme se diversifie, mais les thèmes de voyage: culturels, sportifs, affaires, se banalisent dès qu ils rentrent dans la moulinette des quelques tours opérateurs internationaux.

L'industrie touristique fuit l'originalité et l'innovation, en opposition avec une société mondiale de plus en plus diversifice et hétérogène dans ses habitudes et modes de vie.

Il faudrait coller à la société, l'explorer sous toutes ses facettes et continuer à inventer et collecter des idées, des sujets.

"L'intelligence est aussi un produit d'appel" déclare Pascale Breugnot, productrice à Antenne 2.

L'avenir du tourisme exige tout un travail de recherche et de réflexion sur la façon pour l'individu de communiquer avec le public, de l'associer à une démarche de voyage, de rencontre, de le rendre actif par rapport aux thèmes proposés et de lui donner le sentiment d'avoir acquis quelque chose chemin faisant.

Le voyage, c'est une démarche, pas une recette. Une ambiance. Une multitude de couleurs. Au voyagiste d'animer et de mettre nos imaginations au service de ces couleurs.

Le tourisme des prochaines années doit aider le voyageur à réflecchir sur le monde dans lequel il vit. $80 \%$ des Japonais sont attirés par la culture européenne et tous manifestent une grande curiosité vis-à-vis de la culture française. "Songez-y" conseille Fujio Yoshida, vice-président de Suntory, numéro un mondial du commerce des spiritueux.

\section{Quelque chose de plus...}

La qualité, entrainant les choix de la clientèle, deviendra rapidement un élément de rentabilité. Comment tempếrer une standardisation du produit touristique? En aménageant des éléments de personnalisation, en créant "un climat", en développant le "goût".

Ce "quelque chose de plus..." faisant plus appel à un esprit qu'à des moyens financiers est pour Mare Chevallier, un des responsables d'un centre de formation aux métiers de I'Hồtellerie (Crear) en France, "une des réponses possibles à l'attente des clientèles, dont les exigences vont croissantes et qui, de ce fait, glissent peu à peu vers le secteur commercial", On pourrait ajouter secteur commercial, banal, sans ẩme. Alors que le voyage doit assurer à tous ses acteurs une meilleure insertion dans la société mondiale, un plus grand épanouissement dans leur vie professionnelle. $f$

\section{Références}

(1) LEVITT, Theodore, L imagination au service du Marketing. Théodore Levitt, Revue Economica, 1986.

(2) Voir article de JOPPE, Marion, Le Tourisme Interngtional en 1985 et début 1986 - Espaces, no 83, décembre 1986 .

(3) MALLON, Pierre, Le fourisme Francais a l'ejpreuve, Revue Espaces, février 1987.

(4) BARAE, Josquin, Travailler gutrement, Revue Espaces, août 1995

(5) CONSEIL NATIONAL DU TOURISME, Introduction des nouvelles technolgoies dans le tourisme, 1985.

(6) BARRE, Josquin: article précité.

(7) Rappelons qu'3 peine $8 \%$ des Francais utilisent los services professionnels du woyage pour confectionner leurs vacances.

(8) Le chauffeur Now Yorkais veut retrouver sa pizza et son "hamburger" au Club de Fort en France et n'est pas intéressé par les célebres buffets pantagruéliques, tant appréciés par la clientẻle européenne.

(9) Rapport fait a la CEE IForecasting and assessment in the field of science and technologyl,

(10) PIVIN, J.L., Bureau d'Ingénierie Culturalle de la Fète et dest Lolsirs, Forum Canners 1986 sur les Nouveaux equipements de lolsirs, Edition du Moniteur.

(11) DANZIN, André, L'Europe et l'intelligence , Le Monde, 24 avril 1987.

(12) BĖAUD, Michel, Le systême National Mondial hiérarchisé, Edition La Découwerte, Collesction Analga, 1987.

(13) GIZARD, Xavier, Les services et la ressource humaine, Agence Nationale pour le Developpement de l'Education Permanente, Noisy-Le-Grand, 1987. 